\section{Dr. Ajeganova and Dr. Hafström reply}

\section{To the Editor:}

We thank Dr. Kawada for the comments ${ }^{1}$ about our study ${ }^{2}$. We agree that multivariate analysis is indispensable in predictive models of risk factors, and also support the discussion of the applied statistical approach. However, we would clarify that our analyses were developed first to take into account known and measurable confounders such as age, sex, ever-smoking, hypertension, diabetes mellitus, and body weight. As only age, but not the other traditional risk factors, had substantial influence on the cardiovascular disease (CVD) outcome in our study and as the number of CVD events was too small for a meaningful multivariate analysis, we next conducted separate age-adjusted regression analyses. These included rheumatoid arthritis (RA) disease-related variables identified with an effect with $\mathrm{p}<0.10$ in the primary univariate models, i.e., we applied bivariate analyses. Even if there were a limited number of outcomes, we feel confident about the reported associations as the conclusions of both simple and age-adjusted models were sufficiently consistent.

Dr. Kawada points out the need for careful attention to sample size when interpreting the research results. We consider that for studies of limited size it is especially important to describe patients' characteristics, the setting where they were done, and how they were sampled to allow appropriate generalizability from experience with these patients to other situations ${ }^{3}$. However, only studies of a sufficiently large number of individuals will enable conduct of powerful multivariate analyses. Most studies of risk factors for CVD, like those of prognosis, in the general population have included samples of several thousand individuals. Comparable numbers are difficult to achieve when studying RA, but this does not diminish the importance of studying a sufficient sample size.
As discussed by Dr. Kawada, in terms of outcomes and subjects included, our small-size study may provide overestimated or underestimated results ${ }^{4}$. With respect to the limitations mentioned above, we recognize that our results need confirmation in larger RA inception cohorts, which we are currently conducting. We believe, though, that our study might add to better understanding of cardiovascular risk factors and prognosis in patients with early RA.

SOFIA AJEGANOVA, MD; INGIÄLD HAFSTRÖM, MD, Unit of Rheumatology, Department of Medicine, Karolinska Institutet, Karolinska University Hospital, Huddinge, 14186 Stockholm, Sweden. Address correspondence to Dr. Ajeganova;

E-mail: sofia.ajeganova@karolinska.se

\section{REFERENCES}

1. Kawada T. Prognosis factors for cardiovascular events in patients with rheumatoid arthritis: Multivariate analysis is indispensable [letter]. J Rheumatol 2013;40:337.

2. Ajeganova S, de Faire U, Jogestrand T, Frostegård J, Hafström I. Carotid atherosclerosis, disease measures, oxidized low-density lipoproteins, and atheroprotective natural antibodies for cardiovascular disease in early rheumatoid arthritis - An inception cohort study. J Rheumatol 2012;39:1146-54.

3. Landewe R, van der Heijde D. Primer: Challenges in randomized and observational studies. Nat Clin Pract Rheumatol 2007;3:661-6.

4. Richy F, Ethgen O, Bruyere O, Deceulaer F, Reginster J-Y. From sample size to effect size: Small Study Effect Investigation (SSEi). Internet J Epidemiol 2004;1:12.

J Rheumatol 2013;40:3; doi:10.3899/jrheum.121445 